\title{
Patterns of psychopathology and cognition in sex chromosome aneuploidy
}

\author{
Srishti Rau ${ }^{1,2^{*}}$ (0), Ethan T. Whitman², Kimberly Schauder ${ }^{1,2}$, Nikhita Gogate ${ }^{3}$, Nancy Raitano Lee ${ }^{4}$, \\ Lauren Kenworthy ${ }^{1,2}$ and Armin Raznahan ${ }^{2}$
}

\begin{abstract}
Background: Sex chromosome aneuploidies (SCAs) are a collectively common family of genetic disorders that increase the risk for neuropsychiatric and cognitive impairment. Beyond being important medical disorders in their own right, SCAs also offer a unique naturally occurring model for studying X-and Y-chromosome influences on the human brain. However, it remains unclear if (i) different SCAs are associated with different profiles of psychopathology and (ii) the notable interindividual variation in psychopathology is related to co-occurring variation in cognitive ability.

Methods: We examined scores for 11 dimensions of psychopathology [Child/Adult Behavior Checklist (CBCL)] and general cognitive ability [full-scale IQ (FSIQ) from Wechsler tests] in 110 youth with varying SCAs ( $X X Y=41, X Y Y=22$, $X X X=27, X X Y Y=20)$ and 131 typically developing controls $(X X=59, X Y=72)$.

Results: All SCAs were associated with elevated CBCL scores across several dimensions of psychopathology (twosample $t$ tests comparing the euploidic and aneuploidic groups [all $|T|>9$, and $p<0.001$ ]). Social and attentional functioning were particularly sensitive to the carriage of a supernumerary Y-chromosome. In particular, the XYY group evidenced significantly more social problems than both extra-X groups (Cohen's $d$ effect size $>0.5$, Bonferroni corrected $p<.05$ ). There was marked variability in CBCL scores within each SCA group, which generally correlated negatively with $\mathrm{IQ}$, but most strongly so for social and attentional difficulties (standardized $\beta,-0.3$ ). These correlations showed subtle differences as a function of the SCA group and CBCL scale.

Conclusions: There is domain-specific variation in psychopathology across SCA groups and domain-specific correlation between psychopathology and IQ within SCAs. These findings (i) help to tailor clinical assessment of this common and impactful family of genetic disorders and (ii) suggest that dosage abnormalities of $\mathrm{X}$ - and $\mathrm{Y}$-linked genes impart somewhat distinct profiles of neuropsychiatric risk.
\end{abstract}

Keywords: Sex chromosome aneuploidy, Sex chromosomes, Psychopathology, Cognition, Neurogenetic conditions

\section{Introduction}

Sex chromosome aneuploidies (SCA) are a collectively common family of neurogenetic disorders that arise due to carriage of an atypical number of X- and/or Y-chromosomes other than the typical female $(\mathrm{XX})$ or male $(\mathrm{XY})$ complement [1]. This family of disorders encompasses

\footnotetext{
*Correspondence: srrau@childrensnational.org

2 Section on Developmental Neurogenomics, Human Genetics Branch, National Institute of Mental Health, Bethesda, MD, USA

Full list of author information is available at the end of the article
}

several distinct karyotype groups including XXY (Klinefelter), XYY, XXX (Trisomy X), and XXYY syndrome, among others. As detailed below, these conditions are broadly characterized by increased rates of psychopathology and cognitive impairment [2]. Here, we use the term psychopathology to refer to a range of social-emotional and behavioral difficulties reported in SCA that may or may not reach clinically elevated/ diagnostic thresholds. However, to date, studies of psychopathology have tended to focus on one karyotype group or one domain of psychiatric symptomatology at a time. Therefore, few studies original author(s) and the source, provide a link to the Creative Commons licence, and indicate if changes were made. The images or other third party material in this article are included in the article's Creative Commons licence, unless indicated otherwise in a credit line to the material. If material is not included in the article's Creative Commons licence and your intended use is not permitted by statutory regulation or exceeds the permitted use, you will need to obtain permission directly from the copyright holder. To view a copy of this licence, visit http://creativecommons.org/licenses/by/4.0/. The Creative Commons Public Domain Dedication waiver (http://creativeco mmons.org/publicdomain/zero/1.0/) applies to the data made available in this article, unless otherwise stated in a credit line to the data. 
have made direct comparisons of different domains of psychopathology across different SCA groups, or tested if the SCA group modifies relationships between co-occurring variation in psychopathology and cognitive ability. Addressing these gaps in knowledge would help to clarify genotype-phenotype relationships within SCAs, which could in turn inform both clinical and neuroscientific understanding of these disorders.

There is convergent evidence across several studies for greater psychopathology in SCAs as compared to typically developing euploidic controls [1-3]. Conditions with an extra X-chromosome (e.g., Klinefelter's syndrome [47, XXY; KS]; Trisomy X [47, XXX]) have been associated with increased rates of ADHD (with inattention rather than hyperactivity/impulsivity causing greater impairment), executive dysfunction, anxiety, depression, and social difficulties [1,3-7]. Conditions with an extra Y-chromosome (e.g., 47, XYY) have been associated with increased problems with hyperactivity, defiance, conduct problems, social problems, and low frustration tolerance [8-10], with diagnoses of ADHD and conduct problems being most common $[11,12]$. Sex chromosome tetrasomies (e.g., XXYY, XXXY) are substantially rarer than trisomies and appear to be associated with more pronounced behavioral and socio-emotional difficulties [13]. The most prevalent domains of psychopathology in XXYY syndrome include impulsivity, anxiety, temper tantrums, and social difficulties [14-18], with diagnoses of ADHD and mood disorders being most common [19]. Thus, research to date has helped to quantify different aspects of psychopathology in individual SCA groups, and individual domains of psychopathology across multiple SCA groups $[8,20]$. However, we still lack quantitative comparisons of multiple dimensions of psychopathology across several SCAs [10]. This undertaking is important considering the wide range of symptoms and symptom severity observed among individuals both within and across SCA groups. Such approaches have proved fruitful in other clinical contexts (e.g., the CBCL-Dysregulation Profile [CBCL-DP [21-28];]), which strongly motivates pursuing their application in SCA research.

A second need in SCA research is to better understand the nature of associations between psychopathology and cognitive ability. Both domains are altered by SCA, and both show high interindividual variation across patients. However, we lack a detailed characterization of how these two key clinical outcomes are interrelated. With regard to SCA effects on IQ, there is a recognized inverse relationship between supernumerary sex chromosome count and IQ $[29,30]$. Whereas the sex chromosome trisomies (XXY, XYY, and XXX syndromes) are all associated with an $\sim 10$ point reduction in full-scale IQ on average (slightly larger decrements for verbal as compared to performance IQ), reported mean IQs are typically lower in XXYY syndrome [8, 19, 20, 30, 31]. However, there is marked interindividual variability in IQ within all SCA groups [32-34], and it remains unclear how this is related to the co-occurring interindividual variation that has been described for psychopathology. Work in non-SCA groups suggests there can be complex correlations between clinical variation in IQ and psychopathology [35-45], but the few studies addressing this question in SCA have focused on individual domains of psychopathology [8], prompting the clinically important question of whether some psychiatric features are more strongly coupled to cognitive ability than others in SCA.

The current study aimed to begin addressing the open questions above through analysis of previously unpublished data on psychopathology from a multi-karyotype cohort of individuals with SCA. We first sought to directly compare SCA groups to each other across a range of psychopathology domains, hypothesizing that SCA groups would differ in their profiles of psychopathology. We next considered variations in IQ and psychopathology across individuals with SCAs to test if correlations between these two clinical features varied as a function of psychopathological domain, SCA group, or both. We hypothesized that the magnitude of IQ-psychopathology coupling would vary for different domains of psychopathology, as seen in other clinical groups [43], and possibly further as a function of SCA karyotype.

\section{Methods \\ Participants}

One hundred and ten individuals of varying SCA karyotypes $(X X Y=41, X Y Y=22, X X X=27, X X Y Y=20)$ and 131 typically developing euploidic controls (TD; XX = $59, \mathrm{XY}=72$ ) participated in this study, which was conducted at the National Institute of Mental Health (NIMH) Intramural Research Program (Clinical trial reg no. NCT 00001246, clinicaltrials.gov; NIH Annual Report Number, ZIA MH002794-13; Protocol number: 89-M-0006). Participants with SCA were recruited through SCA support organizations and NIMH websites. Typically developing controls were recruited through the NIH Healthy Volunteer office. Inclusion criteria for the SCA groups were (i) a non-mosaic SCA diagnosis confirmed by karyotype testing, and (ii) no history of brain injury or comorbid neurological disorder. Inclusion criteria for typically developing controls were no history of neurological, neurodevelopmental, or psychiatric illness. Data from typically developing controls were primarily included as a benchmark for observed levels of psychopathology and psychopathology-IQ coupling in SCA groups; substantive group comparisons were restricted to SCA groups. Sample demographics are described in Table 1. This study 


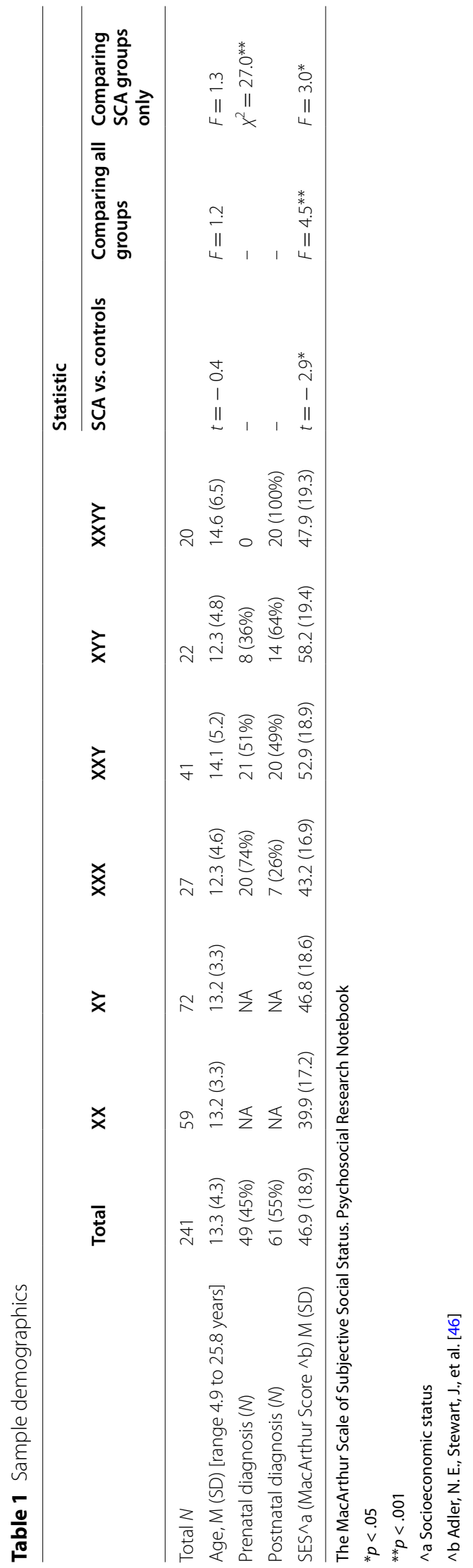


was approved by the NIH Combined Neuroscience Institutional Review Board. All participants and/or their parents provided informed consent or assent, as appropriate. All study protocols were completed at the NIH Clinical Center in Bethesda, Maryland.

\section{Measures}

For the SCA groups, intellectual functioning was estimated using Full Scale IQ (henceforth "IQ") from the Wechsler Intelligence Scale for Children, Fifth Edition (WISC-V [47];) or the Wechsler Adult Intelligence Scale, Fourth Edition (WAIS-IV [48];). For the typically developing groups, IQ was measured using the Wechsler Abbreviated Scale Intelligence, Second Edition (WASI-II, [49]). IQ ratings are expressed as a standard score $(M=$ 100 ; $\mathrm{SD}=15$ ) in comparison to normative expectations based on age. Higher scores indicate higher intellectual functioning.

Psychopathology for all participants was measured using Child Behavior Checklist $(N=219$; CBCL [50];) and Adult Behavior Checklist $(N=22$; ABCL [51];) completed by each participant's caregiver who knew the participant well (e.g., parent). For simplicity, scales from both measures will henceforth be referred to as CBCL subscales, as data were combined across the CBCL and $\mathrm{ABCL}$, and most participants received the CBCL. This widely used instrument combines item-level responses to derive eight syndrome scales (anxious/depressed, withdrawn/depressed, somatic complaints, social problems, thought problems, attention problems, rule-breaking behavior, and aggressive behavior), and three broadband scales: externalizing domain (rule-breaking behavior, aggressive behavior), internalizing domain (anxious/ depressed, withdrawn/depressed, somatic complaints), and total problems (items across all syndrome scales plus an additional 17 items that do not belong to any syndrome scale). Ratings on each scale are expressed as $T$-scores $(M=50$; $\mathrm{SD}=10)$ in comparison to normative expectations based on age and gender. Higher scores indicate the presence of more problem behaviors. For the eight syndrome scales, $T$-scores of 65-69 are borderline clinical, and $T$-scores of 70 or higher are in the clinical range. For the three broadband scales T-scores of 60-63 are borderline clinical and $\mathrm{T}$-scores of 64 or higher are clinically elevated.

\section{Statistical analysis}

We examined the omnibus effect of karyotype group on each of the $11 \mathrm{CBCL}$-derived dimensions of psychopathology using ANOVAs-once considering all groups, and once restricted to just the four SCA groups (applying Bonferroni correction each time). We also used $t$ tests to (i) compare the distribution of CBCL scores in SCA participants (all karyotypes combined) as compared to euploidic controls within our sample (2 sample $t$ tests with Bonferroni correction) and (ii) compare karyotypespecific score distributions for each CBCL scale against the reference norm mean $t$-score of 50 (one sample t-tests with Bonferroni correction). To determine the degree to which any SCA karyotype group effects on CBCL scores existed above and beyond co-occurring variation on general cognitive ability, we also tested for omnibus effects of SCA group on each CBCL dimension while covarying for IQ.

Variation in CBCL subscale scores across SCA groups were visualized using tripartite plots comprising: (i) dot-line plots showing profiles of CBCL subscale scores across SCA groups (Fig. 1A), and profile of SCA group scores across the eight $\mathrm{CBCL}$ syndrome subscales (Fig. 2A), (ii) boxplots showing the distribution of scores per CBCL subscale for each SCA group (Fig. 1B), and scores per SCA group for each CBCL subscale (Fig. 2B), and (iii) heatmaps showing Cohen's $d$ and $p$ values for pairwise t-test comparisons between $\mathrm{CBCL}$ subscales per SCA group (Fig. 1C), and between SCA groups per CBCL syndrome subscale (Fig. 2C). The statistical tests represented by these heatmaps provide a fine-grained analysis of patterned SCA karyotype effects on different dimensions of psychopathology. These comprehensive pairwise t-tests were planned $a$ priori (i.e., not post hoc to omnibus tests) and Bonferroni corrected for multiple comparisons [Fig. 1C: between scales per karyotype, $p$ $=(0.05 / 28)$; Fig. $2 \mathrm{C}$ : between karyotypes per scale, $p=$ $(0.05 / 6)]$.

Finally, to examine potential relationships between psychopathology and cognitive ability in SCA we first used general linear models to predict variation in each of the 11 CBCL subscale scores as a function of IQ, SCA karyotype and the interaction between these two terms. The omnibus $F$-test associated with this interaction term tests the hypothesis that psychopathology-IQ associations vary between SCA groups. In the absence of a significant interaction term, the beta coefficient for the main effect of IQ estimates the relationship between IQ and CBCL subscale scores, controlling for the main effect of the SCA group (Table 2, Fig. 3). To provide a full visualization of all psychopathology-IQ relationships represented in our dataset we generated a matrix of scatterplots between IQ and CBCL scores, faceted by CBCL subscale and SCA karyotype group (Fig. S1). Each pairwise relationship was quantified using the percentage bend robust regression coefficient.

Statistical analyses were performed using $\mathrm{R}$ software [52]), and SPSS Statistics Versions 25 [53] and 26 [54]. The following $\mathrm{R}$ packages were used in data analysis and visualization: ggplot2 [55], and patchwork [56]. 


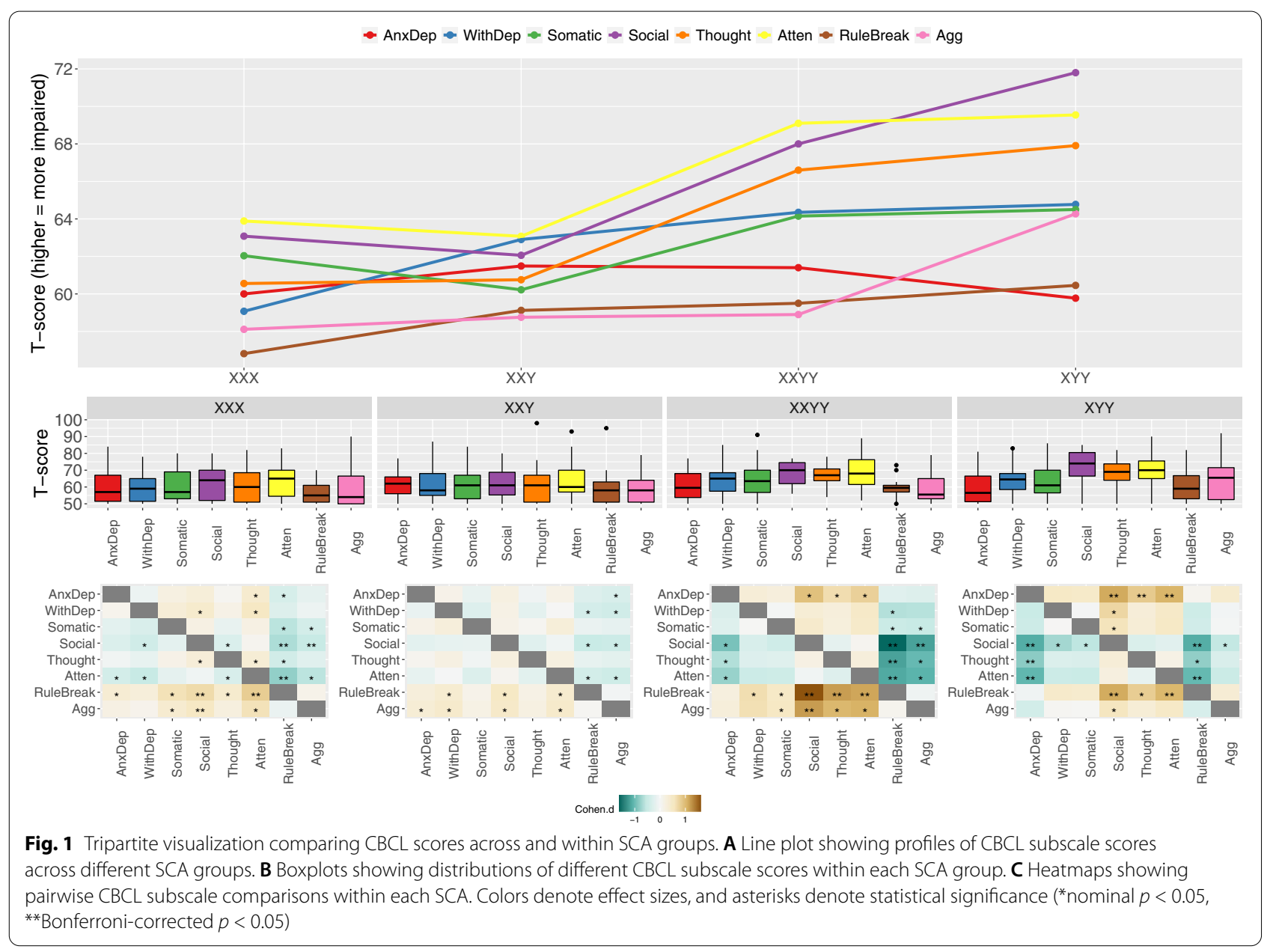

\section{Results}

\section{Profiling SCA effects on psychopathology}

All CBCL subscale scores showed statistically significant differences between the euploidic and aneuploidic groups in two-sample $t$-tests (all $|\mathrm{T}|>9$, and $p<0.001$ ), as well as statistically significant omnibus effect of karyotype in one-way ANOVAs across XX, XY, XXY XYY, XXX, and XXYY groups (all $F \geq 25$ and all $p<.001$, Table 2). We also observed that all subscale scores in all SCA groupswith the notable exception of the externalizing problems broadband scale in XXX syndrome-were significantly elevated above reference norms (one-sample $t$-test against a $T$-score of 50) after Bonferroni correction for multiple comparisons (all corrected $p$ 's <.05).

One-way ANOVAs comparing the four SCA groups alone indicated a statistically significant effect of karyotype group at the uncorrected $p<.05$ level on the following scales: total problems $(F(3,109)=3.8)$, externalizing $F(3,109)=2.8)$, social $F(3,109)=5.6)$, thought $F(3,109)$ $=4.2$ ), and attention problems $F(3,109)=3.0)$ (Table 2). The total problems, and social and thought problems scales survived Bonferroni correction for multiple comparisons across CBCL scales (i.e., $p<.05 / 11$ ). These data support the notion that different SCA karyotypes are associated with partly distinct profiles of psychopathology. The profile of CBCL scores across SCA groups and the profile of SCA group scores across CBCL scales are shown in Figs. 1 and 2A and A, respectively. Accompanying boxplots show $\mathrm{CBCL}$ score distributions by scale within the SCA group (Fig. 1B) and by the SCA group within scale (Fig. 2B).

Pairwise $t$ tests comparing CBCL subscales within SCA karyotype groups (Fig. 1C), and SCA karyotype groups within each CBCL subscale (Fig. 2C) identified the salient contrasts driving variation in CBCL scores across SCA groups (Table 2). Comparison of CBCL subscales within each SCA group (Fig. 2B, C) revealed a relatively even elevation of all subscales in XXY syndrome, while the other three groups each featured specific subscales with notably high or low score elevations. Specifically; the XYY group showed significant elevation of social, thought and attentional problems relative to anxious-depressed and 


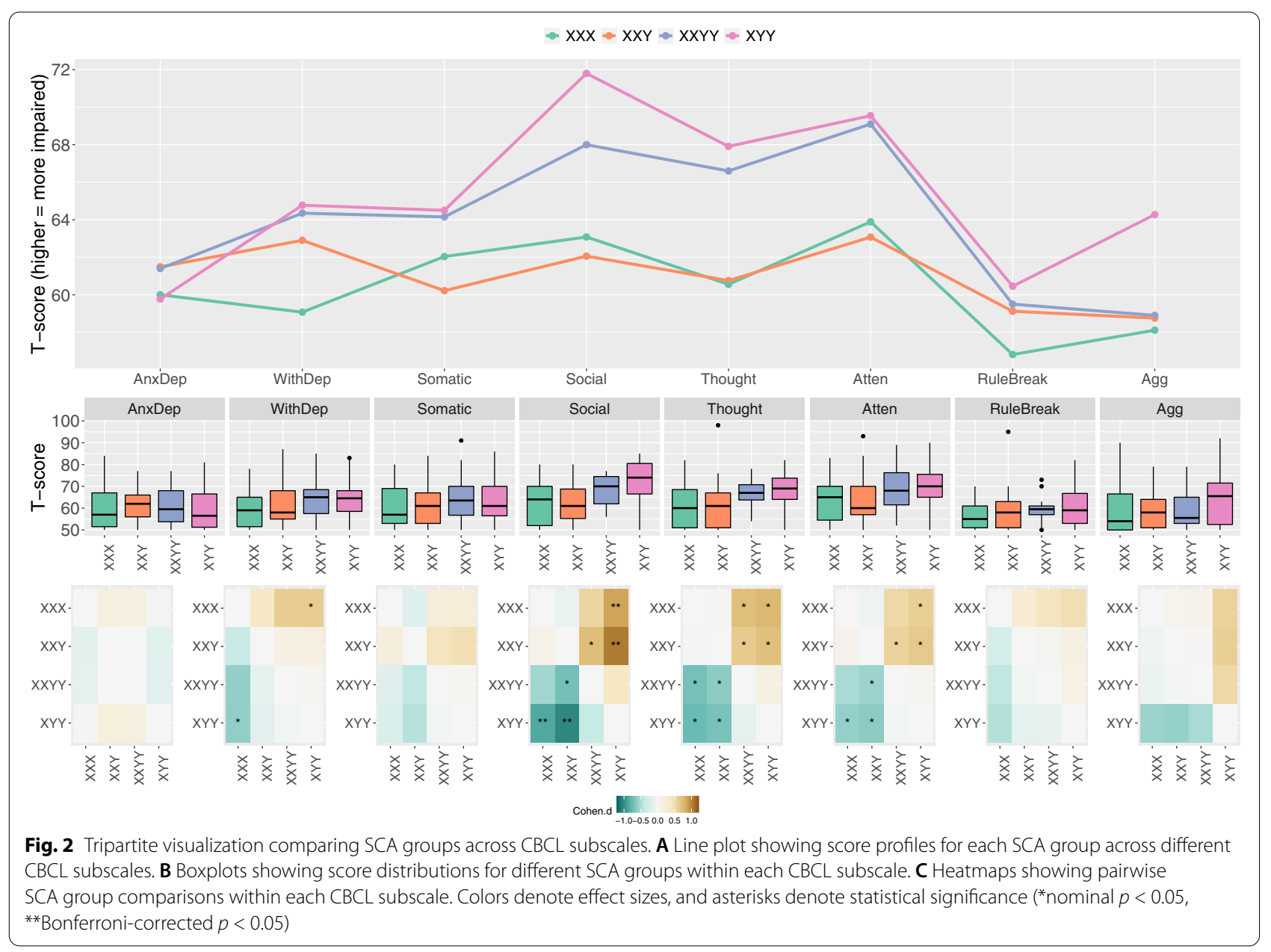

rule breaking problems; the XXYY group showed significant elevation of social, thought and attentional problems relative rule breaking problems, and significant elevation of social problems relative to aggressive behaviors; and the XXX group showed significant elevation of social, and attentional problems relative to rule breaking, and of social problems relative to aggressive behavior (Fig. 1C). Complementing these within-group analyses, comparisons between SCA groups for each CBCL scale indicated that social, thought and attentional problems tended to be more elevated in XYY and XXYY groups as compared to XXY and XX groups (significantly so after Bonferroni correction for social problems). A corollary of the more pronounced impact of XYY and XXYY karyotypes on these domains of psychopathology is that these groups showed the greatest dispersion in mean scores across the measured domains of psychopathology (Table 2, Fig. 1A). Specifically, the CBCL $T$-score standardized deviations (i.e., CBCL score increments of 10) between the highest and lower subscale score in each SCA subgroup were: 1.2 for XYY, 1 for XXYY, 0.7 for XXX, and 0.4 for XXY.
Taken together, the results above indicate that (i) all four of the SCA groups studied are associated with a broad increase in risk for psychopathology, but that social and attentional difficulties tend to be the most strongly impacted subdomains, and (ii) the apparent selective vulnerability of social and attentional domains relative to other aspects of psychopathology is particularly pronounced in SCA groups bearing an additional Y chromosome (XYY and XXYY).

\section{Coupling between IQ and psychopathology in SCAs}

We did not find evidence of statistically significant modulation of IQ-psychopathology relationships by SCA karyotype group (uncorrected $p$-value range: 0.16-0.78 across CBCL subscales). However, given the rarity of this cross-SCA dataset (and associated limitations in statistical power to detect such higher-order interactions), we generated an exploratory visualization of psychopathology-IQ relationships for each unique CBCL subscale-SCA group combination (Fig. S1). 


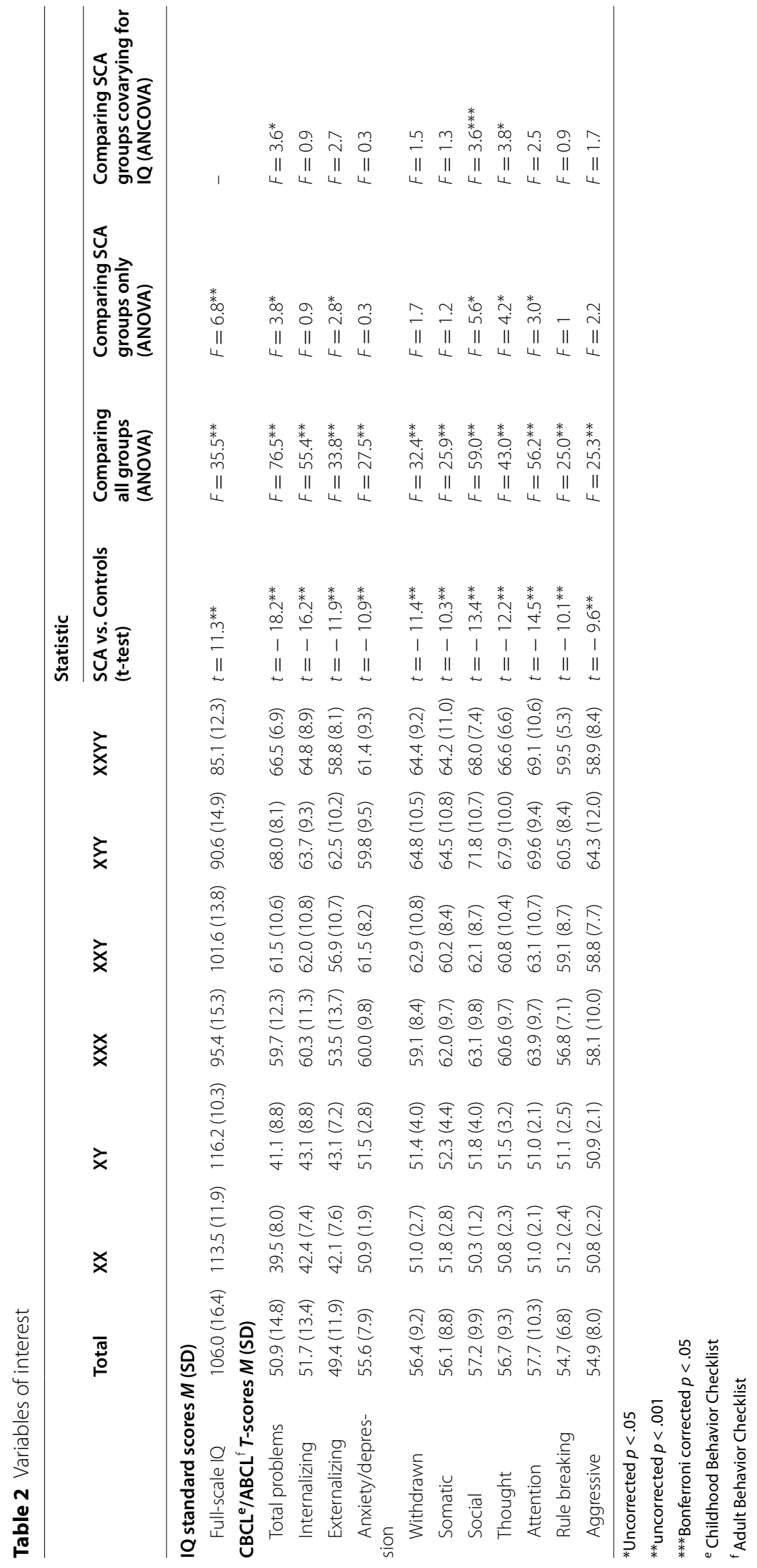




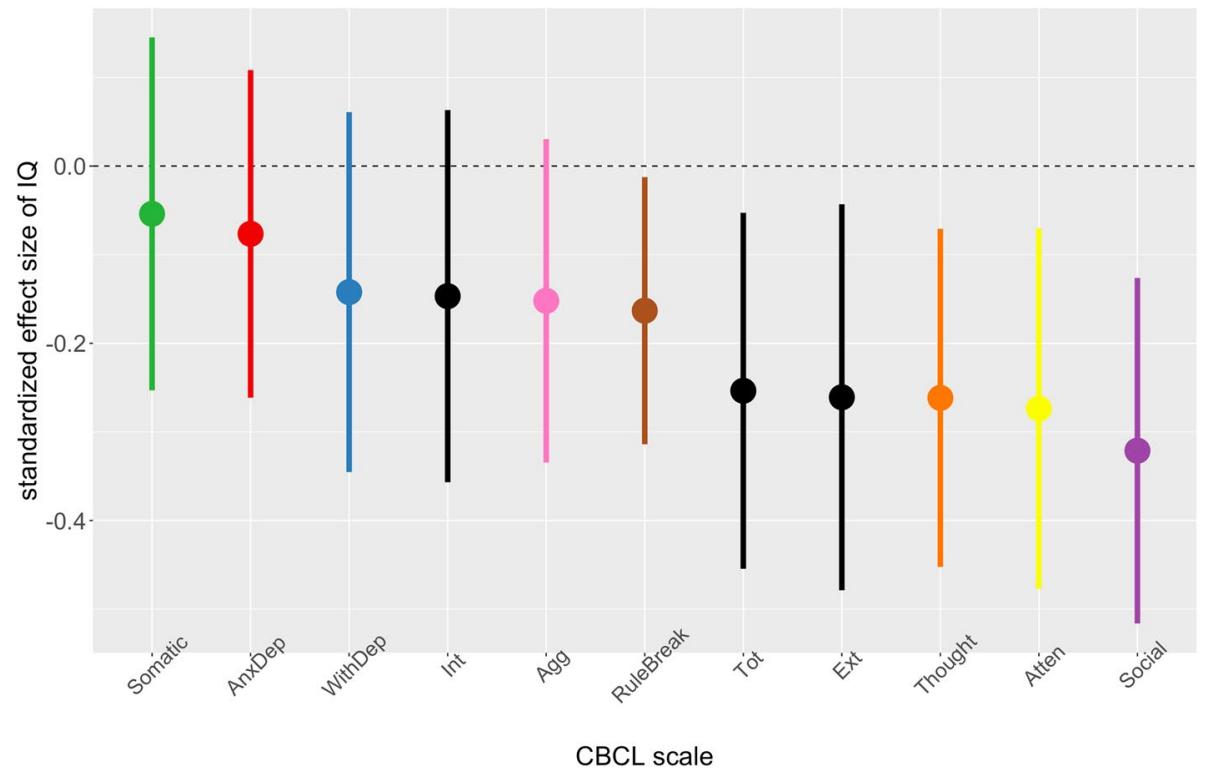

Fig. 3 Associations with IQ for different domains of psychopathology in SCA. For each CBCL scale ( $x$-axis), we provide the estimated standardized regression coefficient for IQ ( $y$-axis: point $=$ estimated coefficient, line $=95 \%$ confidence intervals of coefficient), which estimates the standard deviation shift in $\mathrm{CBCL}$ scores for 1 standard deviation increases in IQ

Qualitatively, this revealed a wide range in estimated correlations between IQ and psychopathology. Specifically, some psychopathology correlations with IQ were moderately negative [e.g., with thought problems in $\mathrm{XXY}(r=-0.48)$, social problems in XYY $(r=-0.47)$, and social problems in XXX $(r=-0.43)]$, while others were near zero (e.g., rule breaking in XXYY, somatic problems in XXX, and thought problems in XYY).

Given the absence of statistically significant modulation of psychopathology-IQ relationships by SCA group (IQ*group interaction Bonferroni-corrected $p$ values > 0.05 for all CBCL subscales), we estimated the standardized regression coefficient for IQ on each CBCL subscale controlling for the main effects of SCA group (Fig. 3). These analyses revealed that across all SCA groups, associations with IQ were strongest for social, attentional, and thought problems (standard deviation [SD] shifts up in psychopathology for 15-point drop in IQ: $0.32,0.27$, and 0.26 respectively), and weakest for domains of somatic problems and anxious-depressed domains (SD shifts up in psychopathology for 15-point drop in IQ both $<0.08$ ). While controlling for IQ, SCA group showed a statistically significant omnibus $F$-test for social problems after correction for multiple comparisons across CBCL scales.

\section{Discussion}

The current study adds to our understanding of psychopathology in SCA in a number of key directions as detailed below.

\section{Broad risk for psychopathology across SCAs}

Our findings in this previously unpublished dataset of behavioral measures in multiple SCA groups add to the mounting consensus that carriage of extra $\mathrm{X}$ - and or Y-chromosomes in humans increases risk for diverse domains of psychopathology [1-3]. Specifically, we observe that almost all domains of psychopathology across all SCA groups were significantly elevated relative to reference norms and study specific controls, suggesting that carriage of extra sex chromosomes results in a broad increase in risk for psychological and behavioral problems across multiple domains. Increases in psychopathology associated with SCA are greatest for measures of total psychopathology, and problems in attentional and social subdomains. These findings reinforce prior reports to underscore the need for standard care in SCAs to be expanded to routinely include psychological screening and referral for treatment as indicated.

\section{Evidence for differential psychopathological risk as a function of SCA karyotype}

We provide new evidence for variation in psychopathology across different domains of measurement and different SCAs. Specifically, our findings point to the clinically important observation that social problems are not only one of the domains of psychopathology that are most impacted by SCAs in general (see above) but is also the domain that varies most between different SCA groups. At a relaxed level of statistical significance (i.e., uncorrected $p<.05$ ), we also find some evidence for variation 
in levels of total psychopathology and externalizing, somatic, attention and thought problems across SCA subgroups. Of note, however, findings for the CBCL thought problems scale should be interpreted with caution as this scale is not necessarily indicative of a thought disorder but also consists of items measuring obsessions, repetitive behaviors, and sleep disturbance [57].

We find that the statistically significant variation in social problems (and to a nominally significant extent, total psychopathology, externalizing, and attentional problems) across SCA groups is driven by higher elevations in groups carrying an extra $\mathrm{Y}$ - as compared to an extra X-chromosome. Thus, carriage of an extra Y-chromosome appears to have a particularly pronounced impact on social and attention problems, as well as problems associated with the CBCL thought problems subscale. These findings are generally consistent with the few existing studies that have compared measures of psychopathology across SCA groups. For example, SCAs defined by carriage of an extra Y-chromosome have shown higher rates of autism spectrum disorder (ASD) and ADHD diagnoses $[10,20,58]$, as well as higher scores on continuous measures of attentional problems [10], as compared to SCAs defined by carriage of an extra X-chromosome. Of note, our data do not support the notion that problems with externalizing and aggressive behaviors are more pronounced in SCAs defined by supernumerary Y- vs. supernumerary X-chromosomes [1, 3, 4, 59]. This lack of statistically significant group differences is associated with weak effect sizes. We therefore interpret this observation as providing further evidence against the previously refuted (yet still actively stigmatizing) notion that carriage of additional Y chromosomes is preferentially associated with aggressive and anti-social behaviors [1]. We also fail to replicate prior reports that carriage of an extra $\mathrm{X}$ chromosome has a greater impact on internalizing symptoms than carriage of an extra Y [3]. Further research in larger cohorts using standardized measures will likely help to resolve these inconsistencies and more definitively resolve the potentially patterned effects of $\mathrm{X}$ - and Y-chromosome aneuploidies on different domains of psychopathology. Nevertheless, we provide evidence that different SCAs may impart slightly different profiles of risk, and that clinical assessment should be attuned to these potential differences.

The potential biological bases for differential Y-vs. $\mathrm{X}$-chromosome effects on social functioning remain unclear. It is notable that disorders of social functioning like ASD and psychopathology are more common in $\mathrm{XY}$ males as compared to XX females in the general population [60], raising the idea that the
Y-chromosome may have a special influence on brain systems subserving social functioning. However, although there is evidence for anatomical changes in social networks within the human brain with Y-chromosome aneuploidy, these same systems are also sensitive to X-chromosome dosage [30,61,62] - which suggests that traditional in vivo measures of regional brain anatomy may not prove useful in identifying markers of the differential psychopathological risks imparted by different SCA karyotypes. It is well established however that the proximal effects of $\mathrm{X}$ - and Y-chromosome aneuploidy on human gene expression are highly divergent [63] (e.g., X- but not Y-dosage effects on genes escaping X-inactivation)-so, there is a pressing need to understand if and how these divergent effects on gene expression might manifest in brain development as a potential source for downstream clinical variation among SCA groups.

\section{Relationship between psychopathology and intellectual functioning in SCA}

To our knowledge, the current study provides the first comparisons of IQ and psychopathology across SCA genotypes and domains of psychopathology. We do not find evidence for statistically significant modulation of IQ-psychopathology relationships as a function of SCA karyotype. However, our observation of varying correlation coefficients (spanning the range $0-|0.48|$ ) in modestly sized dataset hints that different domains of psychopathology may vary in their relationships with IQ as a function of SCA karyotype (Fig. S1). However, there is growing recognition that correlations between different dimensions of cognition and psychopathology can be weak at the population level and require large sample sizes to be reliably estimated [64], which poses a particular challenge for estimating such correlations within individually rare SCA subgroups. Nevertheless, it is notable that some of the relationships between IQ and psychopathology that have been reported in other datasets (autism spectrum disorder [65];, and anxiety [66];)-were not apparent in SCA. Thus, there remains an open question as to whether relationships between IQ and psychopathology show substantial variation between different clinical subgroups.

Across our full SCA cohort, when controlling for the main effect of the SCA group, we find that different domains of psychopathology can vary in the strength of their relationship with IQ, with social and attentional problems being most strongly coupled to IQ variation. This observation is important from a clinical perspective as it recommends particularly close assessment of cognition in individuals with SCA showing pronounced social and/or attentional problems, and vice versa. From 
a biological perspective, the close coupling between socio-attentional and general cognitive deficits suggests that these two domains of functioning may share closely overlapping brain substrates. This notion is supported by emerging evidence that general cognitive functioning is closely tied to functional organization of specific cortical networks (default mode and dorsal attentional) that are also key components of social and attentional systems within the brain [67].

\section{Limitations and future directions}

Our findings should be considered in light of several caveats and limitations. First, it is well recognized that a considerable proportion of individuals carrying extra sex chromosomes remain undetected. As such studies of clinically identified cohorts such as ours may provide inflated estimates of penetrance due to ascertainment bias $[3,68,69]$. Such inflation is likely to be less for SCA subgroups with more severe presentations (i.e., XXYY vs. XXY for example [19]) - providing a potential methodological source for differing presentations between clinically ascertained groups. However, we detect substantial differences in psychopathology between SCA subgroups with similar rates of under-detection (e.g. XXX and XXY [69])-suggesting that karyotype-specific ascertainment biases cannot be the sole driver of our observed subgroup differences. Expansion of SCA cohort sizes in future research will enable adequately powered statistical comparisons of carriers diagnosed prenatally vs those diagnosed postnatally, which can help to provide a proxy for potential ascertainment biases [70].

Larger cohorts will also facilitate characterization of rare SCA tetra- and pentasomy groups (e.g., XXYY and $\mathrm{XXXXY}$ respectively). Indeed, the future study of larger, and more karyotypically diverse SCA cohorts will enable statistical modeling of ordinal X- and Y-chromosome dosage effects on behavioral functioning/psychopathology, intellectual functioning, and relationships between the two, as opposed to categorical group-based comparisons. Such an approach could also include a more mechanistic examination of the factors that mediate the path between genotype and behavioral phenotype through studying variability in intermediate biological phenotypes (e.g., neuroimaging-derived) across karyotypes as potential mediators of discrepant profiles of psychopathology in SCA. Second, our dataset is cross-sectional in nature, and the low population prevalence of SCAs complicates the collection of the large longitudinal datasets that would be required to model age-varying psychopathology. An important goal for future work will be to better understand how the impact of SCA on mental health may vary across the lifespan. Third, although our study considers multiple domains of psychopathology across multiple SCA groups, there is a pressing need for even more multidimensional measurement of clinical features in SCA, including the many continuous (e.g., temperament) and categorical (e.g., psychiatric diagnoses) variables that were not included in our report. Identifying how variability in these dimensions can impact responsiveness to social/behavioral interventions, at the individual and group level, is also an important undertaking for future research.

\section{Conclusion}

Notwithstanding the above caveats, our study builds on prior work to provide a more detailed understanding of the patterning of psychopathology across different SCA groups, and how variation in psychopathology within SCAs related to co-occurring variation in general cognitive ability. Our findings may help to better target clinical assessments of affected individuals and inform thinking about potential biological factors that might organize the patterning of neuropsychiatric difficulties in X- and Y-chromosome aneuploidies.

\section{Abbreviations \\ SCA: Sex chromosome aneuploidy; ADHD: Attention deficit/hyperactivity disorder; KS: Klinefelter syndrome; IQ: Intelligence quotient; TD: Typically developing; NIH: National Institutes of Health; WISC: Wechsler Intelligence Scale for Children; WAIS: Wechsler Adult Intelligence Scale; WASI: Wechsler Abbreviated Scale of Intelligence; CBCL: Childhood Behavior Checklist; ABCL: Adult Behavior Checklist; ANOVA: Analysis of variance; ASD: Autism spectrum disorder; SD: Standard deviation.}

\section{Supplementary Information}

The online version contains supplementary material available at https://doi. org/10.1186/s11689-021-09407-9.

Additional file 1: Figure S1. Exploratory Visualization of Psychopathology-IQ Relationships by SCA Group and CBCL scale. Each point is a person, and IQ-CBCL score associations are shown for each unique SCA group$\mathrm{CBCL}$ scale combination. Fit lines are from general linear models, and provided correlation coefficients are percentage bend robust regression coefficients.

Additional file 2: Supplementary Table 1. Demographic variables.

\section{Acknowledgements}

The authors would like to thank the participants and their families for taking part in this study.

\section{Authors' contributions}

SR, EW, KS, LK, and AR conceived of the study design. NG and NL collected the data. SR and AR conducted the analyses. All authors prepared and reviewed the manuscript. All authors read and approved the final manuscript.

Funding

This research was funded by the NIMH Intramural Research Program.

\section{Availability of data and materials}

The datasets generated and/or analyzed during the current study are not publicly available to protect individual patient privacy. Data are available upon reasonable request. 


\section{Declarations}

Ethics approval and consent to participate

This study was approved by the $\mathrm{NIH}$ Combined Neuroscience Institutional Review Board. All participants and/or their parents provided informed consent or assent, as appropriate.

\section{Consent for publication}

Not applicable.

\section{Competing interests}

The authors declare that they have no competing interests.

\section{Author details}

${ }^{1}$ Center for Autism Spectrum Disorders and Division of Neuropsychology, Children's National Health System, Washington DC, USA. ${ }^{2}$ Section on Developmental Neurogenomics, Human Genetics Branch, National Institute of Mental Health, Bethesda, MD, USA. ${ }^{3}$ The Department of Biochemistry \& Molecular Medicine, The George Washington University Medical Center, Washington DC, USA. ${ }^{4}$ Department of Psychology, Drexel University, Philadelphia, PA, USA.

Received: 28 May 2021 Accepted: 15 November 2021 Published online: 15 December 2021

\section{References}

1. Leggett V, Jacobs P, Nation K, Scerif G, Bishop DVM. Neurocognitive outcomes of individuals with a sex chromosome trisomy: $X X X, X Y Y$, or XXY: a systematic review. Dev Med Child Neurol. 2010;52:119-29.

2. Hong DS, Reiss AL. Cognitive and neurological aspects of sex chromosome aneuploidies. Lancet Neurol. 2014;13:306-18.

3. van Rijn S. A review of neurocognitive functioning and risk for psychopathology in sex chromosome trisomy $(47, X X Y, 47, X X X, 47, X Y Y)$. Curr Opin Psychiatry. 2019;32:79-84

4. Linden MG, Bender BG. Fifty-one prenatally diagnosed children and adolescents with sex chromosome abnormalities. Am J Med Genet. 2002;110:11-8.

5. Tartaglia NR, Howell S, Sutherland A, Wilson R, Wilson L. A review of trisomy X (47,XXX). Orphanet J Rare Dis. 2010;5:8.

6. Bruining $H$, Swaab $H$, Kas $M$, van Engeland $H$. Psychiatric characteristics in a self-selected sample of boys with Klinefelter syndrome. Pediatrics. 2009;123:e865-70.

7. Lenroot RK, Blumenthal JD, Wallace GL, Clasen LS, Lee NR, Giedd JN. A case-control study of brain structure and behavioral characteristics in 47,XXX syndrome. Genes Brain Behav. 2014:841-9. https://doi.org/10. 1111/gbb.12180.

8. Cordeiro L, Tartaglia N, Roeltgen D, Ross J. Social deficits in male children and adolescents with sex chromosome aneuploidy: a comparison of XXY, XYY, and XXYY syndromes. Res Dev Disabil. 2012;33:1254-63.

9. Geerts M, Steyaert J, Fryns JP. The XYY syndrome: a follow-up study on 38 boys. Genet Couns. 2003;14:267-79.

10. Ross JL, Roeltgen DP, Kushner H, Zinn AR, Reiss A, Bardsley MZ, et al. Behavioral and social phenotypes in boys with 47,XYY syndrome or 47,XXY Klinefelter syndrome. Pediatrics. 2012;129:769-78.

11. Ratcliffe SG, Field MAS. Emotional disorder in XYY children: four case reports. J Child Psychol Psychiatry. 1982:401-6. https://doi.org/10.1111/j. 1469-7610.1982.tb00086.x.

12. Visootsak J, Graham JM. Social function in multiple $X$ and $Y$ chromosome disorders: XXY, XYY, XXYY, XXXYs. Dev Disabil Res Rev. 2009:328-32. https://doi.org/10.1002/ddrr.76.

13. Tartaglia N, Ayari N, Howell S, D'Epagnier C, Zeitler P. 48,XXYY, 48,XXXY and 49,XXXXY syndromes: not just variants of Klinefelter syndrome. Acta Paediatr. 2011:100:851-60.

14. Srinivasan R, Wolstencroft J, Erwood M, Raymond FL, Bree M, Hall J, et al. Mental health and behavioural problems in children with XXYY: a comparison with intellectual disabilities. J Intellect Disabil Res. 2019;63:477-88.

15. Schlegel RJ, Aspillaga MJ, Neu R, Gardner LI. Studies on a boy with XXYY chromosome constitution. Pediatrics. 1965;36:113-9.
16. Sørensen $K$, Nielsen J, Jacobsen P, Rølle T. The 48 , XXYY syndrome. J Ment Defic Res. 1978;22:197-205.

17. Fryns JP, Kleczkowska A, Kubień E, Van den Berghe H. XYY syndrome and other $Y$ chromosome polysomies. Mental status and psychosocial functioning. Genet Couns. 1995;6:197-206.

18. Hanley AP, Blumenthal JD, Lee NR, Baker EH, Clasen LS, Giedd JN. Brain and behavior in 48, XXYY syndrome. Neuroimage Clin. 2015;8:133-9.

19. Tartaglia N, Davis S, Hench A, Nimishakavi S, Beauregard R, Reynolds A, et al. A new look at XXYY syndrome: medical and psychological features. Am J Med Genet A. 2008;146A:1509-22.

20. Tartaglia NR, Wilson R, Miller JS, Rafalko J, Cordeiro L, Davis S, et al. Autism spectrum disorder in males with sex chromosome aneuploidy: XXY/Klinefelter syndrome, XYY, and XXYY. J Dev Behav Pediatr. 2017;38:197-207.

21. Mick E, Biederman J, Pandina G, Faraone SV. A preliminary meta-analysis of the child behavior checklist in pediatric bipolar disorder. Biol Psychiatry. 2003;53:1021-7.

22. Holtmann M, Buchmann AF, Esser G, Schmidt MH, Banaschewski T, Lauch M. The Child Behavior Checklist-Dysregulation Profile predicts substance use, suicidality, and functional impairment: a longitudinal analysis: CBCLDP long-term outcome. J Child Psychol Psychiatry. 2011:52:139-47 Wiley.

23. Schroeder J, Weiss J, Bebko J. CBCL profiles of children and adolescents with Asperger syndrome: a review and pilot study. yorkspace-new.library. yorku.ca; 2011; Available from: https://yorkspace-new.library.yorku.ca/ xmlui/handle/10315/33099

24. Bruining H, Eijkemans MJ, Kas MJ, Curran SR, Vorstman JA, Bolton PF. Behavioral signatures related to genetic disorders in autism. Mol Autism. 2014,5:11.

25. Berona J, Horwitz AG, Czyz EK, King CA. Psychopathology profiles of acutely suicidal adolescents: associations with post-discharge suicide attempts and rehospitalization. J Affect Disord. 2017:209:97-104.

26. Guerrera S, Menghini D, Napoli E, Di Vara S, Valeri G, Vicari S. Assessment of psychopathological comorbidities in children and adolescents with autism spectrum disorder using the Child Behavior Checklist. Front Psychiatry. 2019;10:535.

27. Katsuki D, Yamashita H, Yamane K, Kanba S, Yoshida K. Clinical subtypes in children with attention-deficit hyperactivity disorder according to their child behavior checklist profile. Child Psychiatry Hum Dev. 2020;51:969-77.

28. Chawner SJRA, Doherty JL, Anney RJL, Antshel KM, Bearden CE, Bernier $\mathrm{R}$, et al. A genetics-first approach to dissecting the heterogeneity of autism: phenotypic comparison of autism risk copy number variants. Am J Psychiatry. 2021;178:77-86.

29. Linden MG, Bender BG, Robinson A. Sex chromosome tetrasomy and pentasomy. Pediatrics. 1995;96:672-82.

30. Raznahan A, Lee NR, Greenstein D, Wallace GL, Blumenthal JD, Clasen LS, et al. Globally divergent but locally convergent X-and Y-chromosome influences on cortical development. Cereb Cortex. 2016;26:70-9.

31. Tartaglia NR, Ayari N, Hutaff-Lee C, Boada R. Attention-deficit hyperactivity disorder symptoms in children and adolescents with sex chromosome aneuploidy: $X X Y, X X X, X Y Y$, and $X X Y Y$. J Dev Behav Pediatr. 2012;33:309-18.

32. Joseph L, Farmer C, Chlebowski C, Henry L, Fish A, Mankiw C, et al. Characterization of autism spectrum disorder and neurodevelopmental profiles in youth with XYY syndrome. J Neurodev Disord. 2018;10:30.

33. Wilson KE, Fish AM, Mankiw C, Xenophontos A, Warling A, Whitman E, et al. Modeling familial predictors of proband outcomes in neurogenetic disorders: initial application in XYY syndrome. J Neurodev Disord. 2021;13:12.

34. Printzlau F, Wolstencroft J, Skuse DH. Cognitive, behavioral, and neural consequences of sex chromosome aneuploidy. J Neurosci Res. 2017:95:311-9.

35. Carlson CL, Lahey BB, Neeper R. Direct assessment of the cognitive correlates of attention deficit disorders with and without hyperactivity. J Psychopathol Behav Assess. 1986;8:69-86.

36. Dietz KR, Lavigne JV, Arend R, Rosenbaum D. Relation between intelligence and psychopathology among preschoolers. J Clin Child Psychol. 1997:26:99-107.

37. Weiser M, Reichenberg A, Rabinowitz J, Knobler HY, Lubin G, Yazvitzky R, et al. Cognitive performance of male adolescents is lower than controls across psychiatric disorders: a population-based study. Acta Psychiatr Scand. 2004;110:471-5. 
38. Vitacco MJ, Neumann CS, Jackson RL. Testing a four-factor model of psychopathy and its association with ethnicity, gender, intelligence, and violence. J Consult Clin Psychol. 2005;73:466-76.

39. Howlin P, Rutter M, Berger M, Hemsley R, Hersov L, Yule W. Treatment of autistic children. Wiley series on studies in child psychiatry. Oxford:Wiley; 1987. p. 299. Available from: https://psycnet.apa.org/fulltext/1988-97184-000.pdf

40. Anderson J, Williams S, McGee R, Silva P. Cognitive and social correlates of DSM-III disorders in preadolescent children. J Am Acad Child Adolesc Psychiatry. 1989;28:842-6.

41. Pianta RC, Castaldi J. Stability of internalizing symptoms from kindergarten to first grade and factors related to instability. Dev Psychopathol. 1989;1:305-16.

42. Pianta RC, Caldwell CB. Stability of externalizing symptoms from kindergarten to first grade and factors related to instability. Dev Psychopathol. 1990;2:247-58.

43. Keyes KM, Platt J, Kaufman AS, McLaughlin KA. Association of fluid intelligence and psychiatric disorders in a population-representative sample of US adolescents. JAMA Psychiatry. 2017;74:179-88.

44. Abramovitch A, Anholt G, Raveh-Gottfried S, Hamo N, Abramowitz JS. Meta-analysis of intelligence quotient $(\mathrm{IQ})$ in obsessive-compulsive disorder. Neuropsychol Rev. 2018;28:111-20.

45. Sánchez de Ribera O, Kavish N, Katz IM, Boutwell BB. Untangling intelligence, psychopathy, antisocial personality disorder, and conduct problems: a meta-analytic review. Eur J Personal. 2019;33:529-64 SAGE Publications Ltd.

46. Adler N, Stewart J. The MacArthur Scale of Subjective Social Status. 2007. http://www.macses.ucsf.

47. Wechsler D. WISC-V: technical and interpretive manual. Bloomington: Pearson; 2014.

48. Wechsler D. WAIS-IV administration and scoring manual. San Antonio: Psychological Corporation; 2008.

49. Wechsler D. Wechsler Abbreviated Scale of Intelligence-Second Edition (WASI-II). San Antonio: NCS Pearson; 2011.

50. Achenbach TM, Edelbrock C. Child behavior checklist. Burlington, VT. 1991;7:371-92.

51. Achenbach TM, Rescorla L. Manual for the ASEBA Adult Forms \& Profiles: For Ages 18-59: Adult Self-report and Adult Behavior Checklist: ASEBA; 2003.

52. R Core Team. R: A language and environment for statistical computing. Vienna: R Foundation for Statistical Computing; 2017

53. IBM Corp. IBM SPSS Statistics for Windows, Version 25.0. Armonk: IBM Corp; 2017.

54. IBM Corp. IBM SPSS Statistics for Windows, Version 26.0. Armonk: IBM Corp; 2019.

55. Wickham H. Programming with ggplot2. In: Wickham H, editor. ggplot2: elegant graphics for data analysis. Cham: Springer International Publishing; 2016. p. 241-53.

56. Pedersen TL. The composer of plots [R package patchwork version 1.1.1]. Comprehensive R Archive Network (CRAN); 2020; Available from: https:// cran.r-project.org/web/packages/patchwork/index.html [cited 9 Apr 2021]

57. Pandolfi V, Magyar Cl, Dill CA. An initial psychometric evaluation of the CBCL 6-18 in a sample of youth with autism spectrum disorders. Res Autism Spectr Disord. 2012;6:96-108.

58. Bishop DVM, Jacobs PA, Lachlan K, Wellesley D, Barnicoat A, Boyd PA, et al. Autism, language and communication in children with sex chromosome trisomies. Arch Dis Child. 2011;96:954-9.

59. Bender BG, Harmon RJ, Linden MG, Robinson A. Psychosocial adaptation of 39 adolescents with sex chromosome abnormalities. Pediatrics. 1995:96:302-8.

60. Werling DM, Geschwind DH. Sex differences in autism spectrum disorders. Curr Opin Neurol. 2013;26:146-53.

61. Mankiw C, Park MTM, Reardon PK, Fish AM, Clasen LS, Greenstein D, et al. Allometric analysis detects brain size-independent effects of sex and sex chromosome complement on human cerebellar organization. J Neurosci. 2017; $37: 5221-31$

62. Nadig A, Reardon PK, Seidlitz J, McDermott CL, Blumenthal JD, Clasen LS, et al. Carriage of supernumerary sex chromosomes decreases the volume and alters the shape of limbic structures. eNeuro. 2018;5. https://doi.org/ 10.1523/ENEURO.0265-18.2018.
63. Raznahan A, Parikshak NN Chandran V Blumenthal JD, Clasen LS, Alexander-Bloch AF, et al. Sex-chromosome dosage effects on gene expression in humans. Proc Natl Acad Sci U S A. 2018;115:7398-403.

64. Owens MM, Potter A, Hyatt C, Albaugh M, Thompson WK, Jernigan T, et al. Recalibrating expectations about effect size: a multi-method survey of effect sizes in the ABCD study. 2020. Available from: psyarxiv.com/tn9u4

65. Edirisooriya $M$, Dykiert $D$, Auyeung $B$. IQ and internalising symptoms in adolescents with ASD. J Autism Dev Disord. 2020. https://doi.org/10. 1007/s10803-020-04810-y.

66. van Steensel FJA, Heeman EJ. Anxiety levels in children with autism spectrum disorder: a meta-analysis. J Child Fam Stud. 2017;26:1753-67.

67. Feilong M, Guntupalli JS, Haxby JV. The neural basis of intelligence in fine-grained cortical topographies. Elife. 2021;10. https://doi.org/10.7554/ eLife.64058.

68. Gunther DF, Eugster E, Zagar AJ, Bryant CG, Davenport ML, Quigley CA. Ascertainment bias in Turner syndrome: new insights from girls who were diagnosed incidentally in prenatal life. Pediatrics. 2004;114:640-4.

69. Bardsley MZ, Kowal K, Levy C, Gosek A, Ayari N, Tartaglia N, et al. 47,XYY syndrome: clinical phenotype and timing of ascertainment. J Pediatr. 2013;163:1085-94.

70. Berglund A, Stochholm K, Gravholt CH. The epidemiology of sex chromosome abnormalities. Am J Med Genet C: Semin Med Genet. 2020;184:202-15.

\section{Publisher's Note}

Springer Nature remains neutral with regard to jurisdictional claims in published maps and institutional affiliations.

Ready to submit your research? Choose BMC and benefit from:

- fast, convenient online submission

- thorough peer review by experienced researchers in your field

- rapid publication on acceptance

- support for research data, including large and complex data types

- gold Open Access which fosters wider collaboration and increased citations

- maximum visibility for your research: over $100 \mathrm{M}$ website views per year

At BMC, research is always in progress.

Learn more biomedcentral.com/submissions 